\title{
Female Preponderance in the Discordant Association between Retinopathy and Nephropathy in Patients with Type 2 Diabetes Mellitus
}

\author{
Satsuki Kawasaki ${ }^{1,6}$, Shinobu Satoh ${ }^{2,6}$, Osamu Hasegawa ${ }^{3}$, Satoshi Morita ${ }^{4}$, Satoshi Kato ${ }^{5}$, \\ Yasuo Terauchi ${ }^{6}$
}

\begin{abstract}
${ }^{1}$ Department of Endocrinology and Metabolism, Chigasaki Tokushukai Medical Center, Chigasaki, Japan; ${ }^{2}$ Department of Endocrinology and Metabolism, Chigasaki Municipal Hospital, Chigasaki, Japan; ${ }^{3}$ Department of General Medicine, Yokohama City University Medical Center, Yokohama, Japan; ${ }^{4}$ Department of Biostatistics and Epidemiology, Yokohama City University Medical Center, Yokohama, Japan; ${ }^{5}$ Department of Ophthalmology, University of Tokyo School of Medicine, Tokyo, Japan; ${ }^{6}$ Department of Endocrinology and Metabolism, Yokohama City University Graduate School of Medicine,Yokohama, Japan.

Email: satsukik@med.email.ne.jp
\end{abstract}

Received October $19^{\text {th }}, 2010$; revised December $4^{\text {th }}, 2010$; accepted December $10^{\text {th }} 2010$.

\begin{abstract}
Aim: To elucidate clinical features in patients with type 2 diabetes with advanced retinopathy but without nephropathy. Methods: This study examined 1324 patients (784 males and 540 females) with type 2 diabetes mellitus. Diabetic retinopathy was graded according to the International Clinical Classification of Diabetic Retinopathy as no diabetic retinopathy, mild or moderate non-proliferative diabetic retinopathy, severe non-proliferative diabetic retinopathy, and proliferative diabetic retinopathy. Diabetic nephropathy was classified into four stages of severity according to the Guideline Committee of the Japan Diabetes Society. Each patient was examined for retinopathy grade and nephropathy stage. Clinical features of patients with proliferative diabetic retinopathy were compared with regard to the four grades of diabetic nephropathy. Results: Fifty-two patients with type 2 diabetes (3.9\% of the whole series of 1324 patients with type 2 diabetes and $25.7 \%$ of patients with proliferative diabetic retinopathy) had proliferative diabetic retinopathy without the presence of nephropathy. Multiple statistical analysis using a proportional odds model revealed that patients with proliferative diabetic retinopathy without nephropathy had a significantly lower systolic blood pressure $(p<$ $0.001)$ than those who did and were preponderantly female $(p<0.05)$. Conclusions: A possible susceptibility of diabetic females to proliferative advanced retinopathy without nephropathy encourages further studies on the role of hormones and blood coagulation in the pathogensis of proliferative diabetic retinopathy.
\end{abstract}

Keywords: Discordance, Female, Gender preponderance, Hormone, Nephropathy, Pathogenesis, Proliferative, Retinopathy, Risk factor, Type 2 diabetes

\section{Introduction}

Randomized controlled trials have provided evidence that intensive blood glucose control and tight blood pressure control delay the onset and prevent the progression of microvascular complications in patients with type 1 and type 2 diabetes mellitus [1-3]. Specifically, longterm poorly controlled blood glucose levels and systemic hypertension have been implicated in major microvascular complications such as retinopathy and nephropathy, with concordance of the two relatively common $[4,5]$.
However, Agardh et al. [6] reported that 35\% of their diabetic patients with proliferative retinopathy did not have nephropathy and suggested that the underlying etiology of diabetic microvascular complications differs between retinopathy and nephropathy. Chavers et al. [7] reported that several cases of advanced diabetic retinopathy had normal urinary albumin excretion, low-level microalbuminuria, and normal glomerular structure measures. Kanauchi et al. [8] reported that $4.1 \%$ of patients with type 2 diabetes with advanced diabetic neph- 
ropathy did not have concomitant retinopathy to demonstrate a discordant association between retinopathy and nephropathy. These reports indicate inconsistency in the severity of diabetic microvascular complications, but the underlying mechanism for the discordant association between the 2 complications has not been defined. Most previous reports on the association between retinopathy and nephropathy examined patients with type 1 diabetes mellitus, and few studies have examined a large series of patients with type 2 diabetes mellitus. It, therefore, would be useful to study patients with type 2 diabetes mellitus presenting with discordance between retinopathy and nephropathy to improve our understanding of the risk factors of diabetic microvascular complications. We examined a large series of patients with type 2 diabetes mellitus and defined the clinical features of those with proliferative retinopathy without nephropathy.

\section{Materials and Methods}

Studied were a series of 1324 consecutive patients (784 males and 540 females) with type 2 diabetic mellitus who were hospitalized between 1998 and 2005 in the third Department of Internal Medicine, Yokohama City University, and Chigasaki Tokushukai Medical Center. Subjects with relatively severe disease were excluded from this study. Patient characteristics were as follows: age, $59.8 \pm 12.6$ years (mean \pm S.D.); diabetes duration, 125.7 \pm 105.7 months; body mass index (BMI), $24.0 \pm 4.3$ $\mathrm{kg} / \mathrm{m}^{2}$; systolic blood pressure (SBP), $133.6 \pm 20.4 \mathrm{~mm}$ Hg; diastolic blood pressure (DBP) , $77.6 \pm 16.1 \mathrm{~mm} \mathrm{Hg}$; and $\mathrm{HbA} 1 \mathrm{c}, 8.6 \pm 2.2 \%$.

Diabetic retinopathy was assessed by ophthalmologists and graded according to the International Clinical Classification of Diabetic Retinopathy [9] into 4 stages: no diabetic retinopathy, mild or moderate non-proliferative diabetic retinopathy, severe non-proliferative diabetic retinopathy, and proliferative diabetic retinopathy. Severity of diabetic nephropathy was classified according to the Japan Diabetes Society Guidelines [10] as follows: stage 1, albumin-to-creatinine ratio (ACR) $<30 \mathrm{mg} / \mathrm{g}$ creatinine; stage II, ACR $\geqq 30 \mathrm{mg} / \mathrm{g}$ and $<300 \mathrm{mg} / \mathrm{g}$ creatinine (i.e., microalbuminuria); stage IIIA, ACR $\geqq 300 \mathrm{mg} / \mathrm{g}$ creatinine and/or persistent proteinuria with creatinine clearance $\geqq 60 \mathrm{ml} / \mathrm{min}$; and Stage IIIB to Stage V, creatinine clearance $<60 \mathrm{ml} / \mathrm{min}$. First, retinopathy grade was compared with nephropathy stage to determine if there was a correlation in the stage of progression between retinopathy and nephropathy. Secondly, cases with proliferative diabetic retinopathy were grouped as follows according to their stage of nephropathy: stage I nephropathy, P1; stage II nephropathy, P2; stage IIIA nephropathy, P3; and stage IIIB nephropathy or higher, P4. A statistical analysis of the 4 groups was performed for variables that included age, diabetes duration, BMI, systolic blood pressure, HbA1c, and gender ratio.

All data are expressed as mean value \pm S.D. The relationship between any two variables was analyzed by standard correlation analysis using the software StatView, version 5.0 (SAS, Inc., Cary, NC, USA). Analyses of variance were applied for group comparisons, followed by Student't-test for confirmation of statistical associations. The relationship between nephropathy stage in proliferative retinopathy and other relevant covariates was determined by multiple regression analysis and a proportional odds model. Correlation in the stage of progression between retinopathy and nephropathy was tested by regression analysis. Statistical significance was assumed at $P$ values $<0.05$.

This study project was approved by the Research Ethics Committee, Yokohama City University (\#6-7). Informed consent was obtained from each study patient.

\section{Results}

Comparison of the stage of progression of retinopathy with that of nephropathy showed that cases without retinopathy mostly had nephropathy stage 1 (no overt kidney disease) (67.4\%); those with mild or moderate non-proliferative diabetic retinopathy mostly had nephropathy stage 1 (no overt kidney disease) or stage 2 (microalbuminuria) $(71.8 \%)$; and those with proliferative diabetic retinopathy had nephropathy stage IIIB or higher and had decreased creatinine clearance (32.3\%) (Figure 1). Statistical analysis showed a significant correlation in the progression between retinopathy and nephropathy ( $\mathrm{p}$ $<0.001)$.

However, 52 of the 1324 subjects in the series (3.9\%)

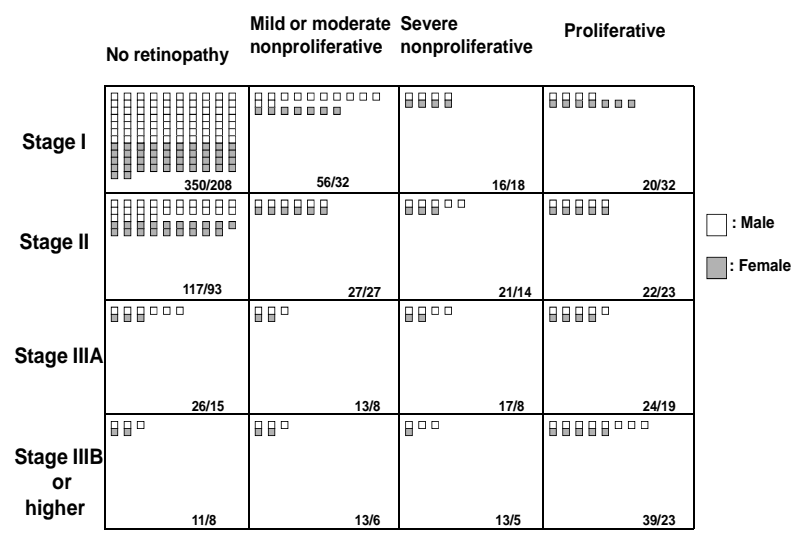

Figure 1. Relationship between Retinopathy and Nephropathy White squares, males; Gray squares, females. Each square represents 5 patients. 
had proliferative diabetic retinopathy without nephropathy (stage I); these 52 cases accounted for $25.7 \%$ of the 202 cases with proliferative diabetic retinopathy. Table 1 shows clinical information on the four stages of nephropathy, consisting of group P1 (52 cases), P2 (45 cases), P3 (43 cases), and P4 (62 cases). Multiple analyses using a proportional odds model with age, diabetes duration, BMI, systemic blood pressure, HbA1c levels, and gender are shown in Table 2. The data reveal that group P1, with proliferative diabetic retinopathy but without nephropathy, had a significantly lower systolic blood pressure than the other three groups, $\mathrm{p}<0.001$ (group P1: $134.7 \pm$ 17.1 mm Hg; P2: $138.5 \pm 21.6$ mm Hg; P3: $143.9 \pm 20.4$ $\mathrm{mm}$ Hg; P4: $153.7 \pm 25.9 \mathrm{~mm} \mathrm{Hg}$ ). With regard to gender (Table 2), the P1 group had a significant female preponderance, $\mathrm{p}<0.05$ (group P1: male 20/female 32; group P2: male 22/female 23; group P3: male 24 /female 19; group P4: male 39 /female 23.). Other variables, including age, diabetic duration, BMI, and HbA1c levels, did not differ among the four groups.

\section{Discussion}

Concordance is usually present between retinal and renal microvascular complications in patients with diabetes mellitus $[4,5]$. This was supported by results of the present study showing a consistent progression of diabetic retinopathy and nephropathy in many of the study patients. However, it was remarkable that $3.9 \%$ of all study patients and $25.7 \%$ of those with proliferative diabetic retinopathy had proliferative diabetic retinopathy but not nephropathy and that the cases with proliferative retinopathy without overt nephropathy were characterized by a significantly lower systolic blood pressure and female preponderance.

Several studies reported discordance as well as concordance between diabetic retinopathy and nephropathy. Agardh et al. [6] performed a retrospective study of 100 patients with insulin-dependent diabetes mellitus to examine the association between retinopathy and nephropathy and found that a high proportion (35\%) of patients with proliferative retinopathy did not have detectable signs of nephropathy and that nephropathy did not develop during an observation period of up to 9 years among these patients. Their data suggested that factors underlying the development of diabetic retinal and renal microangiopathy might be of different origins pathogenetically. Chavers et al. [7] investigated the relationship between retinal structural lesions and quantitative measures of glomerular structure in 86 patients with insulin-dependent diabetes mellitus and found a marked discordance between retinopathy and nephropathy, as illustrated by patients with normal urinary excretion or low-level microalbuminuria, normal glomerular structural measures, and advanced retinopathy. On the other hand, Kanauchi et al. [8] studied 5 out of 122 patients with advanced nephropathy but without retinopathy, summarizing clinical, therapeutic, biochemical and histological data linked to the nephropathy. The divergence between this advanced nephropathy and the concomitant lack of retinopathy led these authors to hypothesize that

Table 1. Clinical background according to nephropathy stage in patients with type 2 diabetes with advanced retinopathy.

\begin{tabular}{lllcc}
\hline Clinical background & P1(52) & P2(45) & P3(43) & P4(62) \\
\hline Age (y) & $62.6 \pm 11.6$ & $60.1 \pm 9.7$ & $58.3 \pm 12.6$ & $61.7 \pm 11.1$ \\
Gender (M/F) & $22 / 47$ & $24 / 36$ & $28 / 25$ & $57 / 33$ \\
Duration (mo) & $189.5 \pm 120.7$ & $171.8 \pm 92.6$ & $160.8 \pm 83.6$ & $223.9 \pm 112.4$ \\
BMI (kg/m $)$ & $22.4 \pm 3.5$ & $24.2 \pm 4.0$ & $25.3 \pm 3.8$ & $23.3 \pm 2.6$ \\
SBP (mmHg) & $134.7 \pm 17.1$ & $138.5 \pm 21.6$ & $143.9 \pm 20.4$ & $153.8 \pm 25.9$ \\
DBP (mmHg) & $74.7 \pm 11.8$ & $76.3 \pm 15.8$ & $79.2 \pm 14.3$ & $80.9 \pm 19.0$ \\
HbA1c (\%) & $8.5 \pm 1.9$ & $9.0 \pm 1.7$ & $9.3 \pm 2.0$ & $8.1 \pm 2.0$ \\
Neuropathy & & & & \\
motor nerve conduction study (m/s) & $41.4 \pm 6.2$ & $39.0 \pm 4.9$ & $38.1 \pm 5.6$ & $33.9 \pm 8.0$ \\
Sensory nerve conduction velocity (m/s) & $46.1 \pm 6.7$ & $42.5 \pm 7.0$ & $42.0 \pm 4.6$ & $35.4 \pm 9.7$ \\
Smoking (yes/no) & $14 / 38$ & $19 / 26$ & $24 / 19$ & $28 / 34$ \\
Treatment (diet/OHA/insulin) & $2 / 18 / 32$ & $0 / 13 / 32$ & $2 / 14 / 27$ & $3 / 10 / 49$ \\
\hline
\end{tabular}

BMI,body mass index; SBP,systolic blood pressure; DBP,diastolic blood pressure; OHA,oral hypoglycemic agent.

Table 2. Multiple analysis using a proportional odds model with age, duration, body mass index (BMI), systolic blood pressure (SBP), HbA1c and gender as explanatory variables.

\begin{tabular}{cccc}
\hline Variables & Odds ratio & 95\%confidenceinterval & $p$ value \\
\hline Age & 0.999 & $0.973-1.026$ & 0.9503 \\
Duration & 1.002 & $1.000-1.005$ & 0.0954 \\
BMI & 1.032 & $0.955-1.114$ & 0.4250 \\
SBP & 1.027 & $1.014-1.039$ & $<0.001$ \\
HbA1c & 0.907 & $0.792-1.038$ & 0.1560 \\
Gender(F/M) & 0.536 & $0.315-0.912$ & $<0.05$ \\
\hline
\end{tabular}


there are important differences in some aspects of the pathogenesis of retinopathy and nephropathy. Sussa et al. [11] noted that all 5 cases with advanced nephropathy but without retinopathy described by Kanauchi et al. [8] were smokers and suggested that the discordance between the complications is due to environmental factors. Recently, Wolf et al. [12] examined 323 patients with type 1 diabetes mellitus and 1906 patients with type 2 diabetes mellitus and found that many patients with type 2 diabetes with renal abnormalities showed, in contrast to those with type 1 diabetes, no signs of retinopathy and recommended renal biopsy in these patients.

Thus, several investigations have indicated the existence of discordance in the development and progression between diabetic retinopathy and nephropathy, but the underlying factors in the absence of association remain undefined. Data in the present study provide new information that female diabetic patients are more likely to present with proliferative diabetic retinopathy without nephropathy. This is in contrast with the widely prevalent view that there is no significant gender preponderance in the development and progression of diabetic retinopathy [13-15]. Mendesohn et al. [16] suggested protective effects of estrogen on cardiovascular diseases. Gender differences in the development and progression of advanced diabetic retinopathy without associated renal disease, as observed in the present study, might be attributed to hormonal influences in females. Diabetic retinopathy has been shown to progress during pregnancy [17-19]. Diabetic retinopathy has been aggravated due to sex hormone administration, followed by return to baseline after its cessation [20]. Further studies are justified to evaluate the influence of blood coagulation and sex hormones in relation to gender with regard to progression of diabetic retinopathy as well as diabetic duration and blood glucose control to understand the mechanism of progression of diabetic retinopathy.

\section{Acknowledgment}

The authors thank the fellow of the Department of Endocrinology and Metabolism, Yokohama City University Graduate School of Medicine, who participated in the care of the patients in this study.

\section{REFERENCES}

[1] The Diabetic Control and Complications Trial Research Group, "The Effect of Intensive Treatment of Diabetes on the Development and Progression of Long-Term Complications in Insulin-Dependent Diabetes Mellitus,” New England Journal of Medicine, Vol. 329, No. 14, 1993, pp. 977-987. doi:10.1056/NEJM199309303291401

[2] UK Prospective Diabetes Group, “Intensive Blood-Glucose Control with Sulphonylurea or Insulin Compared with
Conventional Treatment and Risk of Complications in Patients with Type 2 Diabetes (UKPDS33)," Lancet, Vol. 352, No. 9131, 1998, pp. 837-853. doi:10.1016/S0140-6736(98)07019-6

[3] UK Prospective Diabetes Group, “Tight Blood Pressure Control and Risk of Macrovascular and Microvascular Complications in Type 2 Daibetes (UKPDS38),” British Medical Journal, Vol. 317, No. 7160, 1998, pp. 703-713.

[4] R. Klein, B. E. Klein, S. E. Moss, M. D. Davis and D. L. DeMets, "The Wisconsin Epidemiologic Study of Diabetic Retinopathy. Prevalence and Risk of Diabetic Retinopathy When Age at Diagnosis is Less than 30 Years," Archives of Ophthalmology, Vol. 102, No. 4, 1984, pp. 520-526.

[5] E. A. Friedman and F. A. L’Esperance, "Diabetic Ren Al-Retinal Syndrome," Archives of Internal Medicine, Vol. 140, No. 9, 1980, pp. 1149-1150. doi:10.1001/archinte.140.9.1149

[6] E. Agardh, G. Tallroth, B. Bauer, U. Cavallin-Sjöberg and C. D. Agardh, "Retinopathy and Nephropathy in Insulin-Dependent Diabetics: An Inconsistent Relationship?” Diabetic Medicine, Vol. 4, No. 3, 1987, pp. 248-250. doi:10.1111/j.1464-5491.1987.tb00873.x

[7] B. M. Chavers, S. M. Mauer, R. C. Ramsay and M.W. Steffes, "Relationship between Retinal and Glomerular Lesions in IDDM Patients,” Diabetes, Vol. 43, No. 3, 1994, pp. 441-446. doi:10.2337/diabetes.43.3.441

[8] M. Kanauchi, T. Kawano, H. Uyama, H. Shiiki and K. Dohi, "Discordance between Retinopathy and Nephropathy in Type 2 Diabetes,” Nephron, Vol. 80, No. 1, 1998, pp. 71-75.

[9] C. P. Wilkison, F. L. Ferris, R. E. Klein, P. P. Lee, C. D. Agardh, M. Davis, D. Dills, A. Kampik, R. Pararajasegaram, J. T. Verdaguer, "Proposed International Clinical Diabetic Retinopathy and Diabetic Macular Edema Disease Severity Scales,” Ophthalmology, Vol. 110, No. 9, 2003, pp. 1677-1682. doi:10.1016/S0161-6420(03)00475-5

[10] The Japan Diabetes Society, "Evidence-based Practice Guideline for the Treatment of Diabetes in Japan, 2nd Edition,” Nankodo, Tokyo, 2007.

[11] A. Sessa, G. Batini, M. Meroni, F. Aqneli, F. Giordano and L. T. Tarelli, "Renal-Ritinal Diabetic Syndrome," Nephron, Vol. 83, No. 3, 1999, pp. 285-286. doi:10.1159/000045529

[12] G. Wolf, N. Muller, A. Mandecka and U. A. Müler, “Association of diabetic retinopathy and renal function in patients with types 1 and 2 diabetes mellitus," Clinical Nephrology, Vol. 68, No. 2, 2007, pp. 81-86.

[13] H. A. Kahn and R. F. Bradley, "Prevalence of Diabetic Retinopathy. Age, Sex, and Duration of Diabetes," British Journal of Ophthalmology, Vol. 59, No. 7, 1975, pp. 345-349. doi:10.1136/bjo.59.7.345

[14] K. M. West, M. M. Ahuja, P. H. Bennett, B. Grab, V. Grabauskas, O. Mateo-de-Acosta, J. H. Fuller, J R. J. arrett, H. Keen, K. Kosaka, A. S. Krolewski, E. Miki, V. Schliack and A. Teuscher, "Interrelationships of Mi- 
croangiopathy, Plasma Glucose and Other Risk Factors in 3583 Diabetic Patients: A Multinational Study,” Diabetologia, Vol. 22, No. 6, 1982, pp. 412-420. doi:10.1007/BF00282582

[15] L. I. Rand, A. S. Krolewski, L. M. Aiello, J. H. Warram, R. S. Baker and T. Maki, "Multiple Factors in the Prediction of Risk of Proliferative Diabetic Retinopathy," New England Journal of Medicine, Vol. 313, No. 23, 1985, pp. 1433-1438. doi:10.1056/NEJM198512053132302

[16] M. E. Mendelsohn and R. H. Karas, "The Protective Effects of Estrogen on the Cardiovascular System," New England Journal of Medicine, Vol. 340, No. 23, 1999, pp. 1801-1811. doi:10.1056/NEJM199906103402306

[17] J. Jervell, N. Moe, J. Skjaeraasen, W. Blystad and K. Egge, "Diabetes Mellitus and Pregnancy: Management and Results at Rikshospitalet, Oslo 1970-1977," Diabetologia, Vol. 16, No. 3, 1979, pp. 151-155. doi:10.1007/BF01219791

[18] H. M. Rodman, L. J. Singerman and L. M. Aiello, "Diabetic Retinopathy: Effects of Pregnancy and Laser Therapy,” Diabetes, Vol. 29, No. 1A, 1980, pp. 1.

[19] J. B. M. Moloney and I. M. Drury, "The Effect of Pregnancy on the Natural Course of Diabetic Retinopathy," American Journal of Ophthalmology, Vol. 93, No. 6, 1982, pp. 745-756.

[20] K. Ogawa, N. Okamoto, Y. Saito, S. Harino, M. Namba and T. Hanafusa, "A Case of Diabetic Retinopathy That Progressed with Sex Hormone Administration," Folia Ophthalmologica Japonica, Vol. 44, No. 15, 1993, pp. 1338-1342. 